\title{
NON-ADDITIVE COMBINING ABILITIES
}

\section{RECIPROCALS AND BACKCROSSES}

\author{
NEIL GILBERT \\ John Innes Institute, Bayfordbury, Hertford \\ and \\ J. L. JINKS
}

A.R.C. Unit of Biometrical Genetics, Dept. of Genetics, University of Birmingham

Received I.ix.63

THrs paper applies the method of Gilbert (1963) to Nicotiana rustica data previously reported by Jinks (1954, 1956) and Jinks and Jones (1958). The data consist of flowering dates (in days) and plant heights (ins.) of parents, $F_{1}, F_{2}$ and backcrosses in each of two years in an $8 \times 8$ diallel cross (including reciprocals). For experimental details, see Jinks (1954, 1956).

Can the concepts of Mendelian genetics be reliably injected into practical analyses of polygenic variation?-the authors cannot agree. Here we attempt a purely statistical description of the data: so the Wolf shall abide with the Lamb. Unhappily we cannot agree about which of us is the Lamb.

\section{ALGEBRA}

This section extends the previous analysis (Gilbert, I963) to cover reciprocal differences. The analysis is now applied to $k(k-1)$ observations $y_{i j}$. Here $y_{i j}$ might come from the cross between the $i$ th parent (as male) and the $j$ th parent (as female); or it might come from the backcross of $(i \times j)$ to $(i)$. The method is the same in either case. The model is

$$
y_{i j}=m+a_{i}+b_{j}+c_{i} d_{j}+\text { remainder }
$$

where $\Sigma a=\Sigma b=\Sigma c=\Sigma d=0$ and one arbitrary restriction must be placed on $\Sigma c^{2}$ or $\Sigma d^{2}$. If we include selfs $y_{i i}$, the analysis becomes that of a complete $k \times l$ table (here $l=k$ ) discussed in the previous paper. However, we shall omit selfs, partly because the genetic model of the previous paper suggested that model (I) should not be applied to selfs and crosses simultaneously, and partly because we wish to keep parents ( $\equiv$ selfs) and crosses statistically independent. The least squares equations are

$$
\begin{aligned}
& (k-2) \sum_{j \neq i} z_{i j} d_{j}=(k-2) c_{i} \Sigma d^{2}-k c_{i} d_{i}{ }^{2}+\Sigma c d^{2}+\frac{d_{i} \Sigma c d}{k-\mathrm{I}} \\
& (k-2) \underset{j \neq i}{\sum} z_{j i} c_{j}=(k-2) d_{i} \Sigma c^{2}-k c_{i}{ }^{2} d_{i}+\Sigma^{2} d+\frac{c_{i} \Sigma c d}{k-\mathrm{I}}
\end{aligned}
$$


where $z_{i j}$ is the "special combining ability" remaining after fitting additive male and female parental constants according to Yates (1947). These equations reduce to the corresponding equation on p. 67 of Gilbert (1963) when $z_{i j}=z_{j i}$ and $c_{i}=d_{i}$ (or $c_{i}=-d_{i}$ with the sign of $z_{i j}$ reversed). Here we no longer need to distinguish between the "positive" and " negative" models (I) and (2) of the previous paper. This fact, coupled with the small differences actually observed between $c_{i}$ and $d_{i}$, removes one possible objection; namely that one diallel cross might contain a section best analysed by the " positive " model, and another section which requires the " negative". All the plant height data opted for the " negative" model for reciprocal sums (and so $c_{i} \simeq-d_{i}$ ), whereas flowering dates all chose the " positive" (i.e. $c_{i} \simeq d_{i}$ ). Since the convergence of the iterative solution of equations (2) is even slower than before, it proved convenient to apply the previous analysis to reciprocal sums and then proceed to a new computer programme for solving equations (2). The sum of squares for multiplicative constants is

$$
\Sigma c_{i} z_{i j} d_{j}=\frac{\mathrm{I}}{k-2}\left[(k-2) \Sigma c^{2} \Sigma d^{2}-k \Sigma c^{2} d^{2}+\frac{(\Sigma c d)}{k-\mathrm{I}}^{2}\right]
$$

with $2 k-3$ d.f. (Our model places 3 constraints on the values of $c_{i}$ and $d_{i}$ : or alternatively, the sums of equations (2a), of equations $(2 \mathrm{~b})$, and of $\left[c_{i}(2 \mathrm{a})-d_{i}(2 \mathrm{~b})\right]$ are all identically zero.) As pointed out before, the analysis is really concerned with the simultaneous fitting of additive and multiplicative constants to $y_{i j}$. In the analyses presented here, the m.s. for additive combining abilities exceed the m.s. for multiplicative c.a. That is merely a consequence of extracting the additive parameters first, and would be reversed if we fitted multiplicative parameters first.

\section{2. $1953 \mathrm{~F}_{1}$ PLANT HEIGHT}

As an example we analyse completely one set of data. There is no reason to suppose that the other sets of data, if analysed in similar detail, would behave differently. For each block and for the sum of the two blocks, the additive-multiplicative model has been fitted first to reciprocal sums, and then distinguishing between reciprocals. All figures in this paper are quoted on a per-plant basis.

As Gilbert (1963) pointed out, m.s. for "multiplicative c.a." cannot validly be tested against error: but here they can be tested against each other. Since the model is not additive, s.s. for " block differences " cannot be found directly from block differences, but must be obtained by subtracting s.s. for " block sums" from s.s. for each block separately. Similarly for "reciprocal differences". Now, an additive model applied to block sums must necessarily be exactly equivalent to the same model applied to each block separately (and then summed over blocks). Such equivalence is no longer automatic 
TABLE I

Analysis of variance of $1953 F_{1}$ plant heights

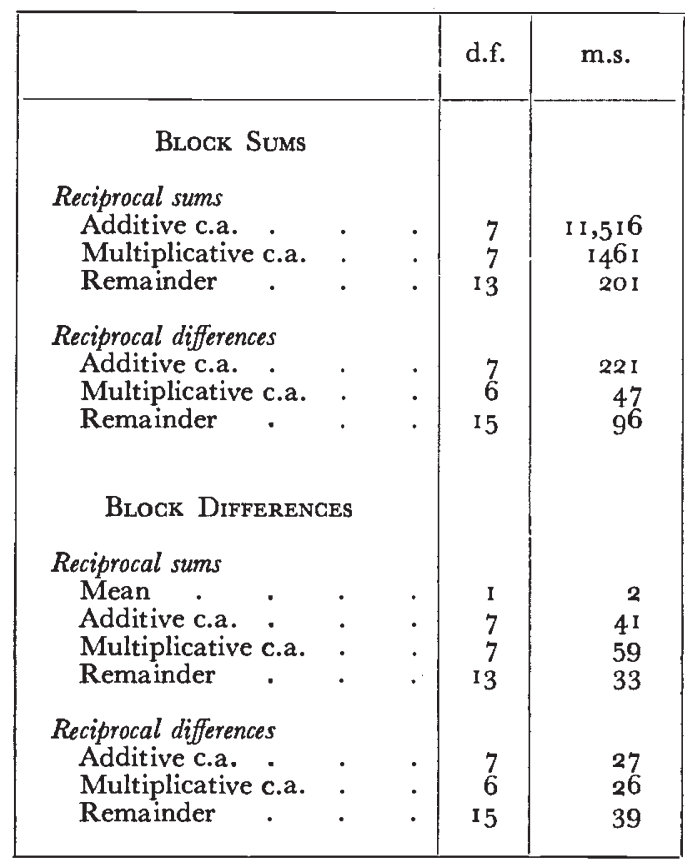

here, and so it is comforting to notice that the multiplicative c.a. do not differ significantly between blocks. Neither do they show reciprocal differences. In section 4 , only the backcross generations are analysed for reciprocal differences.

\section{ANOMALOUS PARENTS}

In two cases ( $1952 \mathrm{~F}_{2}$ plant height and 1952 backcross flowering date) one very large, negative value of $c$ is counterbalanced by a set of positive values. We shall denote the outstanding value by $c_{j}$. Such cases arise when the interactions involving parent $j$ are more important than the remainder. In the extreme we have

$$
\begin{array}{ll}
c_{i}=c+\delta_{i} / c & \text { all } i \neq j) \\
c_{j}=-(k-\mathrm{I}) c+\delta_{j} / c &
\end{array}
$$

where the $\delta$, which are small compared with $c$, sum to zero and are chosen so as to predict exactly the observed interactions involving parent $j$, while a purely additive scheme is predicted for the remaining crosses. In such circumstances, the value of $c$ is arbitrary. The case of $195^{2} F_{2}$ plant height is so close to this extreme that it proved impossible to obtain convergence to a final set of $c_{i}$ s, but easy enough to find any number of sets of values, all of which give effectively the same 
predictions. One of these sets is quoted in table 5. Parents I, 2 and 4 show, in general, the largest absolute values of $c$; and Jinks (1954, I956) interprets the departures from additivity evinced by the crosses involving those parents, as evidence for non-allelic interactions between additive genes. The present analysis is rather different. It not only draws attention to these same parents, but flatly asserts that in $1952 \mathrm{~F}_{2}$ the departures from additivity of parent 4 far outweigh those of any other parent, when examined by this additive-multiplicative model. It is clearly advantageous that the analysis can draw attention to such " anomalous" parents: the technical difficulty over convergence is a consequence of using diallel cross data.

\section{NUMERICAL RESULTS}

"Error" is derived from comparisons between replicates. The remainder m.s. usually exceeds " error ", but only by a factor of about

TABLE 2

Analyses of variance of $F_{1}$ s

\begin{tabular}{|c|c|c|c|c|c|}
\hline & \multirow{3}{*}{ d.f. } & \multicolumn{4}{|c|}{ m.s. for } \\
\hline & & \multicolumn{2}{|c|}{ plant height } & \multicolumn{2}{|c|}{ flowering date } \\
\hline & & $195^{2}$ & 1953 & $195^{2}$ & 1953 \\
\hline Additive c.a. & & & $x 1,5 \times 6$ & 897 & 3408 \\
\hline Multiplicative c.a. & 7 & 581 & $146 I$ & 222 & 922 \\
\hline Remainder & 13 & 144 & 201 & $4 \mathrm{I}$ & 135 \\
\hline Reciprocal differences & 28 & 23 & 117 & 106 & 217 \\
\hline Error & 56 & 26 & 37 & 21 & 22 \\
\hline
\end{tabular}

TABLE 3

Analyses of variance of $F_{2}$ s

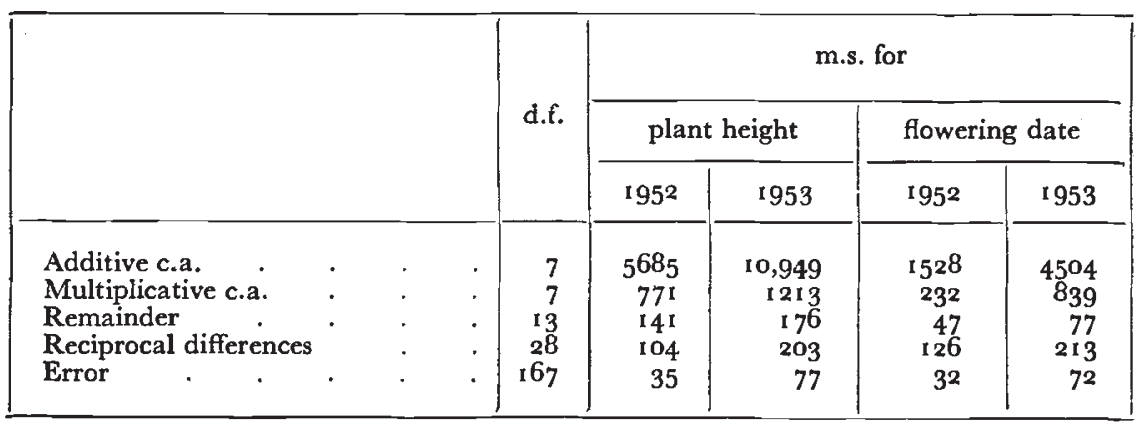

four, so that the additive-multiplicative model describes the data not perfectly, but well enough for any practical purpose. The genetic model discussed by Gilbert ( 1963 ) predicts no reciprocal differences in the backcross multiplicative c.a.; this prediction is not fulfilled. 
TABLE 4

Analyses of variance of backcrosses

\begin{tabular}{|c|c|c|c|c|c|}
\hline & \multirow{3}{*}{ d.f. } & \multicolumn{4}{|c|}{ m.s. for } \\
\hline & & \multicolumn{2}{|c|}{ plant height } & \multicolumn{2}{|c|}{ flowering date } \\
\hline & & 1952 & 1953 & 1952 & I 953 \\
\hline Additive c.a. & 7 & 4955 & 9879 & 1298 & $593^{\circ}$ \\
\hline Additive c.a. $\times$ reciprocals & 7 & 260 & 745 & 979 & 1924 \\
\hline Multiplicative c.a. & 7 & 494 & I 54 & $2 \mathrm{II}$ & 759 \\
\hline Multiplicative c.a. $\times$ reciprocals & 6 & 152 & 249 & 137 & 305 \\
\hline Remainder & 13 & 97 & 192 & 54 & $5^{6}$ \\
\hline Remainder $\times$ reciprocals & i5 & 34 & 64 & I2I & $9 \mathrm{I}$ \\
\hline Error & 109 & 55 & 66 & 40 & 52 \\
\hline
\end{tabular}

TABLE 5

Values of $c$ for plant height ( from $y_{i j}=m+b_{i}+b_{j}-c_{i} c_{j}$ )

\begin{tabular}{|c|c|c|c|c|c|c|c|c|c|}
\hline Parent: & & I & 2 & 3 & 4 & 5 & 6 & 7 & 8 \\
\hline $\begin{array}{l}1952 F_{1} \\
1953 F_{1} \\
1952 F_{2} . \\
1953 F_{2} \\
1952 \text { Backcross } \\
1953 \text { Backcross }\end{array}$ & & $\begin{array}{c}2 \cdot 65 \\
2 \cdot 21 \\
34 \cdot 674 \\
2 \cdot 38 \\
2 \cdot 72 \\
2 \cdot 50\end{array}$ & $\begin{array}{l}-2 \cdot 27 \\
-3 \cdot 74 \\
34 \cdot 621 \\
-1 \cdot 90 \\
-2 \cdot 03 \\
-1 \cdot 41\end{array}$ & $\begin{array}{c}0 \cdot 8 \mathrm{I} \\
0 \cdot 66 \\
34 \cdot 665 \\
1 \cdot 25 \\
0 \cdot 43 \\
1 \cdot 01\end{array}$ & $\begin{array}{r}-3 \cdot 15 \\
-2.67 \\
-242.51 \\
-3.27 \\
-2.21 \\
-3.95\end{array}$ & $\begin{array}{c}-0 \cdot 15 \\
0.94 \\
3 \cdot 637 \\
0 \cdot 49 \\
0 \cdot 33 \\
-0 \cdot 01\end{array}$ & $\begin{array}{c}\mathrm{I} \cdot 45 \\
3 \cdot \mathrm{IO} \\
34 \cdot 635 \\
\mathrm{I} \cdot 62 \\
0.63 \\
\mathrm{I} \cdot 76\end{array}$ & $\begin{array}{c}0.32 \\
-1 \cdot 08 \\
34.637 \\
-0.86 \\
0 \cdot 09 \\
0 \cdot 02\end{array}$ & $\begin{array}{c}0.34 \\
0.57 \\
34.641 \\
0.28 \\
0 \cdot 05 \\
0.08\end{array}$ \\
\hline
\end{tabular}

TABLE 6

Values of $c$ for flowering date ( from $y_{i j}=m+b_{i}+b_{j}+c_{i} c_{j}$ )

\begin{tabular}{|c|c|c|c|c|c|c|c|c|c|c|}
\hline \multicolumn{3}{|c|}{ Parent: } & I & 2 & 3 & 4 & 5 & 6 & 7 & 8 \\
\hline $195^{2}$ & & & -0.96 & 0.63 & -3.91 & 0.35 & $1 \cdot 33$ & 0.06 & $I \cdot I 2$ & $\mathrm{I} \cdot 38$ \\
\hline 1953 & $\mathbf{F}_{1}^{1}$ & & $-1 \cdot 66$ & $\mathrm{I} \cdot 55$ & $-2 \cdot 74$ & $1 \cdot 62$ & I. .64 & $-2 \cdot 77$ & $I \cdot 52$ & 0.83 \\
\hline 1952 & & - & -0.27 & 0.86 & $-3 \cdot 24$ & -0.46 & $\mathrm{I} \cdot 2 \mathrm{I}$ & -0.16 & 0.95 & $I \cdot I I$ \\
\hline 195 & & . & $-2 \cdot 34$ & $0 \cdot 28$ & -0.77 & -0.69 & 3.03 & $-1 \cdot 52$ & 0.20 & $\mathrm{I} \cdot 8 \mathrm{O}$ \\
\hline 1952 & Backcross & . & $-3 \cdot 7^{2}$ & 0.27 & -0.04 & 0.15 & I.33 & -0.06 & $0 \cdot 60$ & $I \cdot 46$ \\
\hline I 953 & Backcross & . & $-2 \cdot 32$ & 0.47 & $-1 \cdot 98$ & -0.97 & $2 \cdot 01$ & -0.12 & I $\cdot 36$ & $\mathrm{I} \cdot 55$ \\
\hline
\end{tabular}

The case of $195^{2} F_{2}$ is discussed in section 3. Otherwise the values of $c$ are very similar, indeed much more so than the values of $z_{i j}$ from which they are estimated.

\section{RELATION BETWEEN GENERATIONS}

Since the values of $b$ and $c$ describe the various crosses sufficiently well, we enquire how to predict them from earlier generations. Here the data are unsatisfactory for several reasons. The number of parents is small: regression co-efficients estimated from only eight points must 
be treated with some reserve, however small their estimated statistical errors may be. The values of $c$ for the cases discussed in section 3 are anomalous. The values of $b$ and $c$ are themselves highly correlated, and since this is certainly not always the case, any observed relation between generations may be peculiar to the present data. But, unlike other published cases, the experiments reported here include backcrosses; we shall concentrate on this aspect.

The genetic model mentioned in section 4 (and indeed any genetic model which postulates additive gene effects, and excludes non-allelic interactions) predicts:-

(I) Equal multiplicative c.a. for $\mathbf{F}_{2}$ and backcross reciprocal sums.

(2) Equal additive c.a. for $F_{2}$ and backcross reciprocal sums.

(3) No reciprocal differences for backcross multiplicative c.a.

(4) Reciprocal difference for backcross additive c.a.

$$
=a_{i}-b_{i} \text { in Model (I), }=\frac{1}{2} p_{i}+\text { constant. }
$$

For (I) and (3) see section 4 . The observed values of $\left(a_{i}-b_{i}\right)$ which, incidentally, are identically equal to the reciprocal differences between the combining abilities of the purely additive model-do not fulfil prediction (4). In fact, Jinks (1956) uses this criterion to test for non-allelic interactions between additive genes.

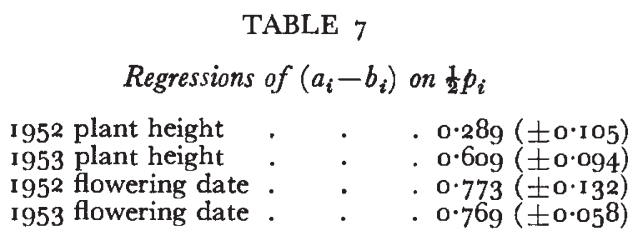

But prediction (2) is much more accurate:

TABLE 8

Correlations ( 6 d.f.) between additive c.a. for $F_{2}$ and backcross reciprocal sums

\begin{tabular}{|c|c|c|c|}
\hline I $95^{2}$ plant height & & & 0.979 \\
\hline I 953 plant height & & & . 0.988 \\
\hline I952 flowering date & & & . 0.941 \\
\hline
\end{tabular}

The $F_{2}$ and backcross additive c.a. are, in fact, equal within the limits of error. The data suggest-but do not prove-that the relation between combining abilities in the same generation ( $F_{2}$ and backcross) is clearer than that between combining abilities in different generations.

\section{DISCUSSION}

What, then, has the analysis achieved? It has shown once again that the additive-multiplicative model can describe rather accurately the crosses in one generation in terms of two parameters per parent, without postulating any particular genetic model. The statistical 
parameters nevertheless have some (unknown) genetical meaning, since their values in successive generations are always more highly correlated than are the observations from which they are estimated. Since the model is purely statistical, prediction from generation to generation can only be empirical; not enough sets of data have yet been analysed for us to decide whether, and how, such prediction can be usefully made. One criterion will evidently be, whether this statistical prediction improves on the prediction obtainable by fitting a genetic model to the data. Genetic models certainly try to probe deeper into the situation, but they cannot help making numerous simplifying assumptions, since the number of parameters must not exceed the number of observations. The choice is between a statistical model with no theoretical basis, and a genetic model with (possibly) too much.

\section{SUMMARY}

An additive-multiplicative statistical model is extended to include reciprocal differences. The model describes the crosses in any one generation remarkably well; but prediction from one generation to the next remains uncertain.

Acknowledgment.-We thank Dr F. Yates, F.R.S., for the use of the Rothamsted computer.

\section{REFERENCES}

GILbert, N. 1963. Non-additive combining abilities. Genet. Res., 4, 65-73.

JINks, J. L. I954. The analysis of continuous variation in a diallel cross of Nicotiana rustica varieties. Genetics, 39, 767-788.

JiNks, J. L. 1956. The $F_{2}$ and backcross generation from a set of diallel crosses. Heredity, 10, I-30.

JiNKs, J. L., AND JONES, R. M. I958. Estimation of the components of heterosis. Genetics, 43, 223-234.

YATES, F. 1947. The analysis of data from all possible reciprocal crosses between a set of parental lines. Heredity, $1,287-30 \mathrm{r}$. 